\title{
Imaging, Intervention, and Workflow in Acute Ischemic Stroke: The Calgary Approach
}

\author{
(D) C. Zerna, (D) Z. Assis, (DC.D. d'Esterre, (D)B.K. Menon, and (D) M. Goyal
} -

\begin{abstract}
SUMMARY: Five recently published clinical trials showed dramatically higher rates of favorable functional outcome and a satisfying safety profile of endovascular treatment compared with the previous standard of care in acute ischemic stroke with proximal anterior circulation artery occlusion. Eligibility criteria within these trials varied by age, stroke severity, imaging, treatment-time window, and endovascular treatment devices. This focused review provides an overview of the trial results and explores the heterogeneity in imaging techniques, workflow, and endovascular techniques used in these trials and the consequent impact on practice. Using evidence from these trials and following a case from start to finish, this review recommends strategies that will help the appropriate patient undergo a fast, focused clinical evaluation, imaging, and intervention.
\end{abstract}

ABBREVIATIONS: MR CLEAN = Multicenter Randomized Clinical trial of Endovascular treatment for Acute ischemic stroke in the Netherlands; EMS = Emergency Medical Services; ESCAPE = Endovascular Treatment for Small Core and Proximal Occlusion Ischemic Stroke; EXTEND-IA = Extending the Time for Thrombolysis in Emergency Neurological Deficits-Intra-Arterial; REVASCAT = Endovascular Revascularization With Solitaire Device Versus Best Medical Therapy in Anterior Circulation Stroke Within 8 Hours; SWIFT-PRIME Solitaire With the Intention for Thrombectomy as Primary Endovascular Treatment

$E^{n}$ ndovascular treatment of acute ischemic stroke in the anterior acirculation due to large-vessel occlusion is now the new standard of care as a result of 5 recently published trials with positive outcomes. Each of the recently published clinical trials (MR CLEAN, ESCAPE, SWIFT-PRIME, EXTEND-IA and REVASCAT) enrolled 70-500 previously independent subjects with (proximal) anterior circulation occlusion. ${ }^{1-5}$ Eligibility varied by age, baseline stroke severity, treatment time window, IV rtPA treatment, and additional extracranial occlusions. A variety of imaging techniques were used to determine the site of occlusion and salvageable brain, including multiphase CTA, CT perfusion, and diffusion-weighted MR imaging. Results clearly favored emergency endovascular intervention in the management of large proximal vessel occlusions in eligible patients in addition to standard care. Endovascular intervention is safe and effective for achieving reperfusion and substantially reduces the degree of disability while increasing the proportion of patients with functional

From the Calgary Stroke Program, Clinical Neurosciences, and Department of Radiology, University of Calgary, Calgary, Alberta, Canada.

Please address correspondence to Mayank Goyal, MD, FRCPC, Seaman Family MR Research Centre, Foothills Medical Centre, 1403 29th St NW, Calgary, AB T2N 2T9 Canada; e-mail: mgoyal@ucalgary.ca

-- Indicates open access to non-subscribers at www.ajnr.org

三 Indicates article with supplemental on-line table.

http://dx.doi.org/10.3174/ajnr.A4610

independence 3 months after stroke (summarized in the On-line Table). ${ }^{1-5}$

These recent endovascular trials led to 4 major conclusions, in our opinion: 1) Fast reperfusion is the key to good outcome ("time is brain”), 2) endovascular treatment by using stent retrievers is safe and effective, 3) imaging plays a critical role in patient selection for endovascular therapy, and 4) teamwork is the key to success.

Herein, we lead the reader along the management workflow of a patient with acute ischemic stroke treated within the ESCAPE trial, from the onset of stroke until the 90-day outcome assessment. We use this actual patient example to illustrate our current approach to acute ischemic stroke management and, more specifically, to endovascular therapy. We discuss this approach in the context of results from the recent endovascular trials. ${ }^{1-5}$

\section{From Acute Ischemic Stroke Onset to 90 Days: A Case Experience}

Stroke treatment is a time-sensitive matter. A treatment goal for acute ischemic stroke is now 60 minutes from hospital arrival to the bolus of IV rtPA. ${ }^{6}$ Specialized stroke centers have successfully reduced their door-to-needle time for IV rtPA to 20 minutes; there is a call for a new benchmark door-to-needle time of $<30$ minutes. ${ }^{7}$ Because the recent endovascular trials demonstrate that fast reperfusion is the key to a good outcome, metrics such as onset-to-reperfusion time and imaging-to-reperfusion time will gain importance. 


\section{Workflow: Improving Preclinical Times}

The success of endovascular treatment relies on timely access to hospitals with fully equipped angiography suites. Patient education will likely ensure fast recognition of stroke syndromes. Subsequent medical contact by phone should lead to priority Emergency Medical Services (EMS) dispatch and transport to the nearest facility for hyperacute treatment. Geographic access to such centers can be challenging. With the "drip and ship" paradigm, patients are initially assessed, imaged, and, if eligible, undergo thrombolysis at a nearby primary stroke center before being transferred to a tertiary stroke center with an angiography suite. In contrast, the "mothership" paradigm leads to direct patient transfer to a tertiary stroke center after field assessment by EMS. These paradigms are mainly influenced by the local health care infrastructure and possibilities for transport. Prior studies have shown that direct referral of patients with stroke from a community hospital without prior imaging before transport shortens the onset-to-treatment time. ${ }^{8,9} \mathrm{~A}$ centralized model of endovascular care with a high-volume dedicated hub hospital receiving patients could help maintaining case volumes, providing expertise and efficient in-hospital systems, and reducing door-to-recanalization times, thereby further improving patient outcomes. ${ }^{10}$ This needs to be balanced with quick access to a primary stroke center and thrombolysis. To achieve this balance, we will need to adapt triage rules and processes and train new and existing personnel.

Our case took place in a city with an angiography suiteequipped hospital.

14:00: Last Seen Healthy. A 71-year-old man with no remarkable medical history aside from being a past smoker was last seen healthy by his wife at 14:00 hours when she left the house to run some errands.

16:14. When she returned $>2$ hours later, she found her husband with right-sided weakness and unable to speak. She called Emergency Medical Services.

16:21. EMS arrived at the patient's home and found the patient sitting in a chair, unable to follow commands or verbally interact. The right arm fell rapidly. The patient was able to walk with a 2-person assist to the ambulance but was dragging his right foot. They determined that he had a major stroke and decided to transport him directly to the comprehensive stroke center.

16:41. They called the triage desk at this hospital and described the patient's symptoms. The triage nurse completed the Short TurnAround Time stroke checklist. The patient fit the Short TurnAround Time stroke criteria of speech disturbance and rapidly falling limbs within 4.5 hours from onset. A Short Turn-Around Time stroke page including expected time of hospital arrival went out to the stroke team, including the emergency department and neurointerventional team, and to the $\mathrm{CT}$ suite.

\section{Workflow: Improving Door-to-Imaging Times}

Hospital arrival to imaging time can be reduced under the following conditions: Prior patient information is available; the patient is transferred directly from door to scanner; and a quick, focused clinical assessment is performed in parallel. Local EMS personnel

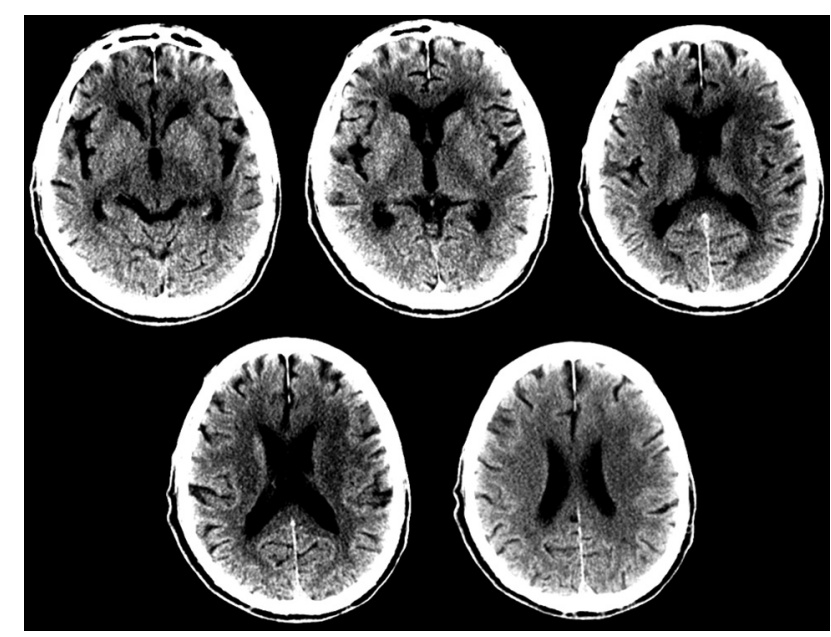

FIG 1. The unenhanced CT ASPECTS score is 7, with 1 point subtracted for early ischemic changes in the caudate nucleus, lentiform nucleus, and insula on the left.

need to be trained to recognize major strokes, and a centralized paging system can prepare emergency staff for patient arrival. ${ }^{11}$

17:09. The patient arrived in the emergency department and was assigned an electronic number for subsequent diagnostic or treatment orders rather than taking all his demographics at the triage desk. In Calgary, we have developed a stretcher-to-CT protocol to minimize delays. The patient stayed on the EMS stretcher and was quickly assessed by the emergency physician. His vital parameters were recorded. The NIHSS score was approximately 29 points. While the patient was being positioned on the CT table, the stroke neurologist acquired additional information about the medical history, current medication, recent surgery, or trauma from the patient's family. In this case, the patient history included no contraindications against IV rtPA or endovascular treatment. Routine blood work was drawn in parallel.

\section{Neuroimaging: Ruling Out Intracranial Hemorrhage}

Because of its fast and widely available acquisition, noncontrast CT remains the primary imaging technique for stroke syndrome presentations. It helps to quickly differentiate ischemic and hemorrhagic stroke and to measure the extent of early ischemic changes with ASPECTS. ASPECTS applies a 10-point scale at the basal ganglia and supraganglionic level of the MCA territory and subtracts 1 point for each subtle parenchymal hypoattentuation of tissue in 1 of the 10 regions. The details of ASPECTS interpretation are available at www.aspectsinstroke.com. All recent clinical trials used NCCT as a first-line imaging tool in most patients. $^{1-5}$

17:25. An NCCT scan for the patient was acquired. There was no sign of intracranial hemorrhage. The ASPECTS score was 7-8 with early ischemic changes in the caudate nucleus, lentiform nucleus, and insula (Fig 1). In Calgary, we have divided the ASPECTS score into 3 broad categories: $0-4$, poor; 5-6, moderate; and 7-10, good. In our opinion, this scoring system is more practical for decision-making.

Immediately after the NCCT, a decision for IV tPA is made, and the bolus is administered to the patient on the CT table 


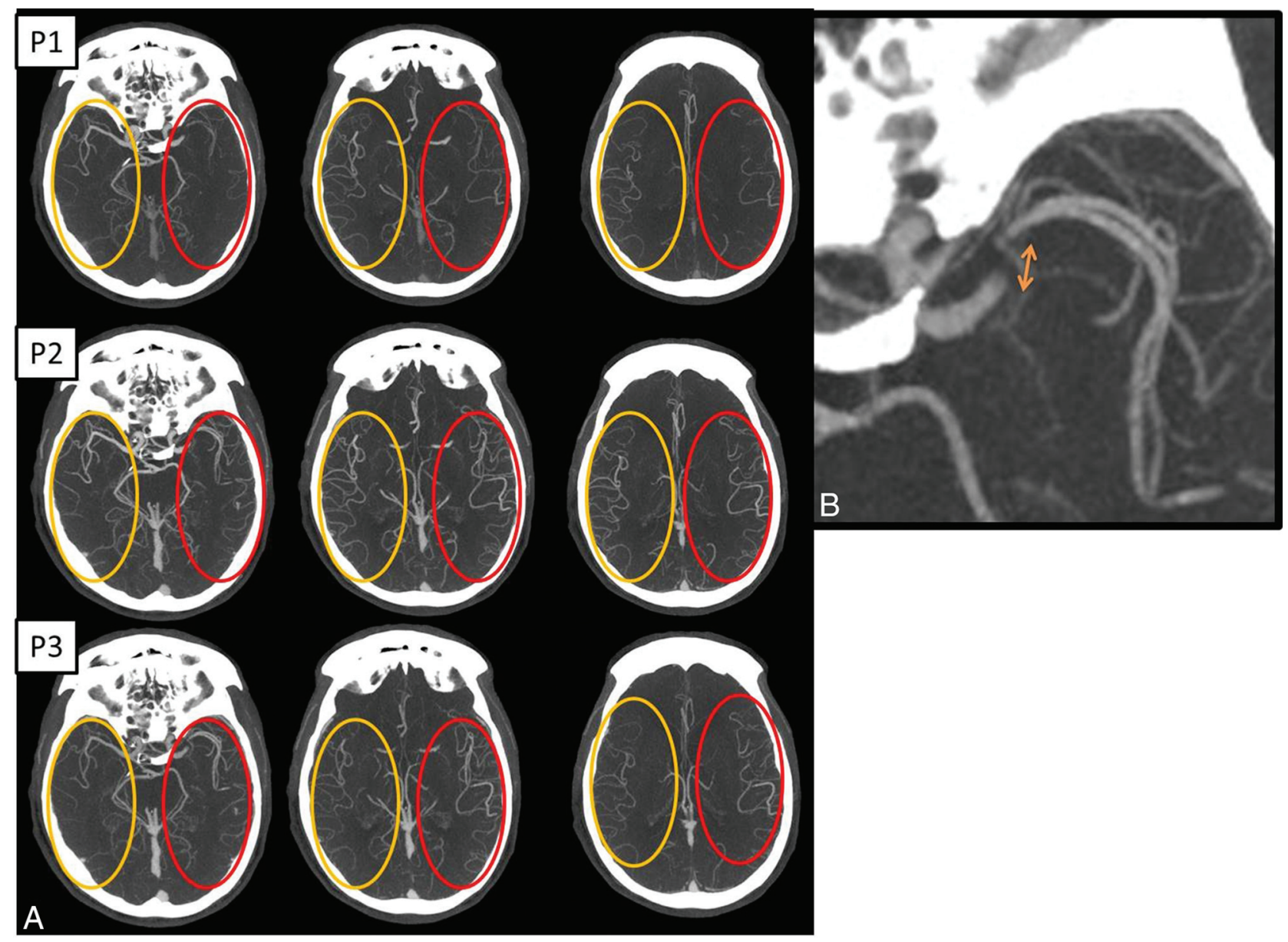

FIG 2. A, Axial multiphase CT angiography (3 phases) maximum-intensity-projection images (arterial, phase 1 [P1]; midvenous, phase 2 [P2]; and late venous, phase 3 [P3] from top to bottom) show left middle cerebral artery segment 1 occlusion. In addition, during phase 1, there is a delay in contrast opacification of the left middle cerebral artery branches compared with the contralateral normal side; however, during phase 2, the contrast opacification appears to be symmetric. This is labeled as "one phase delay." There is also symmetric "extent" of collaterals (red oval) compared with the contralateral normal side (yellow oval). Overall, this gives an impression of fairly good collaterals. B, Magnified axial MIP image in the second delayed phase (P3) shows the possible clot length (arrow) calculated as the distance between the site of abrupt vessel cutoff (proximal end) and the site of distal vessel opacification, either through slow anterograde or retrograde collateral filling (distal end). This can help in preplanning the length of the stent retriever to be used.

(door-to-needle time of 16 minutes), while the CT technician is preparing the patient for the next set of imaging, including CTA (and CT perfusion, if necessary).

\section{Neuroimaging: The Intracranial Occlusion and Collaterals}

To provide images of cerebral blood vessels, a CTA is most commonly used. All recent clinical trials used a CTA technique to detect proximal intracranial occlusions. ${ }^{1-5}$ Additionally, collateral status can be measured by assessing backfilling pial arteries distal to the intracranial occlusion compared with the unaffected contralateral hemisphere. ${ }^{12}$ The quality of collateral assessment depends on the timing of the image acquisition in relation to the IV contrast bolus. If obtained accurately (eg, by using multiphase CTA), collateral status helps to corroborate the status of the brain parenchyma. We typically obtain 2 additional phases of CTA (a total of 3 phases). The ESCAPE trial used collateral imaging to increase the reliability of NCCT ASPECTS assessments. Furthermore, the anatomy of the aortic arch and tortuosity of extracranial vessels and the status of ipsilateral carotid bifurcation, circle of Willis collaterals, and site and size of the thrombus help the neu- rointerventionalist choose the appropriate devices for endovascular treatment. ${ }^{13}$

17:27. The multiphase CTA of this patient showed an occlusion of the M1 segment of the left MCA with the presence of good collaterals (Fig 2A, -B). We additionally went back and looked at the NCCT in light of the findings on the collaterals. This step helps improve the accuracy of the NCCT ASPECTS assessment.

At this stage, because the patient had a proximal occlusion and good collaterals, a decision was made in the CT suite at 17:32 hours to take the patient for endovascular thrombectomy by using stent retrievers. The key components of decision-making available in the CT suite were the following:

1) Clinically severe ischemic stroke (NIHSS 29)

2) Relatively early from onset (212 minutes from last seen healthy)

3) Excellent premorbid status

4) Proximal vessel occlusion: M1

5) Small core/good collaterals on NCCT and multiphase CTA 
6) No major access or technical challenges (eg, severe arch tortuosity).

\section{Neuroimaging: Approximation of Infarct Core/Salvageable Brain Tissue}

CT perfusion has been shown to provide information about tissue salvageability but still remains controversial and therefore not universally adopted. It has many limitations, including lack of standardization, effect of motion, potential for seriously slowing down of the decision-making process, and lack of uniform definitions of core and penumbra and so forth. ${ }^{14}$ Varying studies have used different CTP parameters and thresholds to define ischemic core and penumbra, yet we still do not know the exact threshold for total at-risk tissue and tissue that will die if reperfusion occurs within 20-60 minutes from CT, which is now possible in dedicated centers. There has been a recent move toward fully automated software that allows timely postprocessing of perfusion functional maps robust to common artifacts, allowing rapid clinician interpretation even after hours. ${ }^{15}$

Even in the best of circumstances, the acquisition of CT perfusion data (especially proper z-axis coverage for arterial input function selection), transferring for postprocessing, the postprocessing itself (including motion correction and so froth), and interpretation can consume time. The median time between head CT and successful postprocessing of CTP imaging in the SWIFTPRIME and EXTEND-IA trials was approximately 22 minutes (M. Goyal, personal communication, August, 2015) and 6 minutes 30 seconds, respectively. ${ }^{15}$

17:33. CT perfusion imaging showed a time-to-maximum lesion to indicate ischemic core, while CBV was maintained throughout the area of hypoperfusion (the low CBV ASPECTS was 10). In Calgary, this study was performed as part of a research study. It was not examined acutely and was not used for decision-making.

\section{Neuroimaging: A Decision-Making Paradigm}

In general, the more complicated the imaging technique gets, the more time it takes. The pragmatic approach should be to get by with the minimum necessary information that is relevant for the decision-making process. The decision to pursue thrombectomy is an evolving process, with some factors pushing us toward intervening (moderate-to-severe stroke, age younger than 80 years, potential for independent living, large vessel occlusion, time from symptom onset $<6$ hours) and some arguing against it (an inability to obtain access due to vascular disease, serious comorbidities, patient views on life and disability, living will). These factors help a physician determine when he or she has enough confidence to proceed without the need for further studies (no further test threshold). During this decision-making process, the chance of a poor outcome with thrombectomy increases as time passes and with each additional test obtained. ${ }^{16}$ In Calgary, our decisionmaking approach is based on the following: 1) exclusion of patients with poor ASPECTS, 2) the presence of proximal vessel occlusion, and 3) the absence of poor collaterals on multiphase CTA. We do not typically use CT perfusion for decision-making.

\section{Workflow: Improving Imaging-to-Puncture Time}

In the ESCAPE trial, the key metrics of performance were head CT to groin puncture (target, $<60$ minutes) and head CT to first reperfusion (target, $<90$ minutes). ${ }^{2}$ The start of the NCCT was used to measure this metric. This was deliberately chosen to encourage efficient image acquisition, interpretation, and decisionmaking. This time metric is influenced by the type of imaging technique used, the postprocessing and physician interpretation time, and the efficiency of the image arrival/retrieval system. ${ }^{17}$ During scanning, further information can be acquired from EMS personnel, family members, or electronic patient data bases, if available. The SWIFT-PRIME trial aimed for a qualifying imaging-to-puncture time target of $\leq 70$ minutes, with a permitted maximum of 90 minutes. ${ }^{4}$ If patients are eligible, the workflow around IV rtPA administration can be time-consuming because it is usually administered in a dedicated space away from the angiography suite and sometimes patients are assessed for clinical improvement before being moved to the angiography suite and notification of the endovascular team. ${ }^{18}$ The recent trials proved that endovascular therapy is effective with or without IV rtPA. We do not wait for clinical improvement, and we move the patient as soon as possible to the angiography suite. A group-alert paging system for acute stroke, like we use in Calgary, enables all team members to be prepared and provide enough time to travel to the hospital. Cross-training X-ray and CT technicians to help in the angiography suite may speed up the process on weekends or during nights.

17:47. After obtaining surrogate consent from the patient's wife, the patient was brought to the endovascular suite. In Calgary, the angiography tray is always ready (Brisk Recanalization Ischemic Stroke Kit), and the patient was positioned on the angiography table.

\section{Neurointervention: Use of Anesthesia}

A growing body of evidence is against the use of general anesthesia in acute stroke intervention, with higher rates of poorer outcome and mortality in the general anesthesia group. Hypotension during general anesthesia is also considered as a contributing factor toward a poorer outcome. ${ }^{19}$ The ESCAPE and REVASCAT trials recommended against the use of general anesthesia for endovascular intervention (only 9.1\% in ESCAPE and 6.7\% in REVASCAT). ${ }^{2,5}$ We believe that with the availability of newer generation stent retrievers and prior knowledge of vascular anatomy from CTA, it is possible to achieve successful recanalization despite some degree of patient motion. The stroke physician provides conscious sedation if necessary and helps the interventional team with all aspects of patient management.

\section{Neurointervention: Initiating the Endovascular Procedure}

It is ideal to have a prearranged stroke tray ready for use in the angiogram suite at all times so that the procedure can be initiated without delay. We also emphasize using standardized techniques and devices as much as possible. ${ }^{20}$ Almost all our patients with anterior circulation stroke end up having an $8 \mathrm{~F}$ femoral sheath with an $8 \mathrm{~F}$ balloon-guide catheter (parked in the internal carotid artery; the level is decided on the basis of tortuosity and can usually be determined on the CTA), an appropriate coaxial selective 


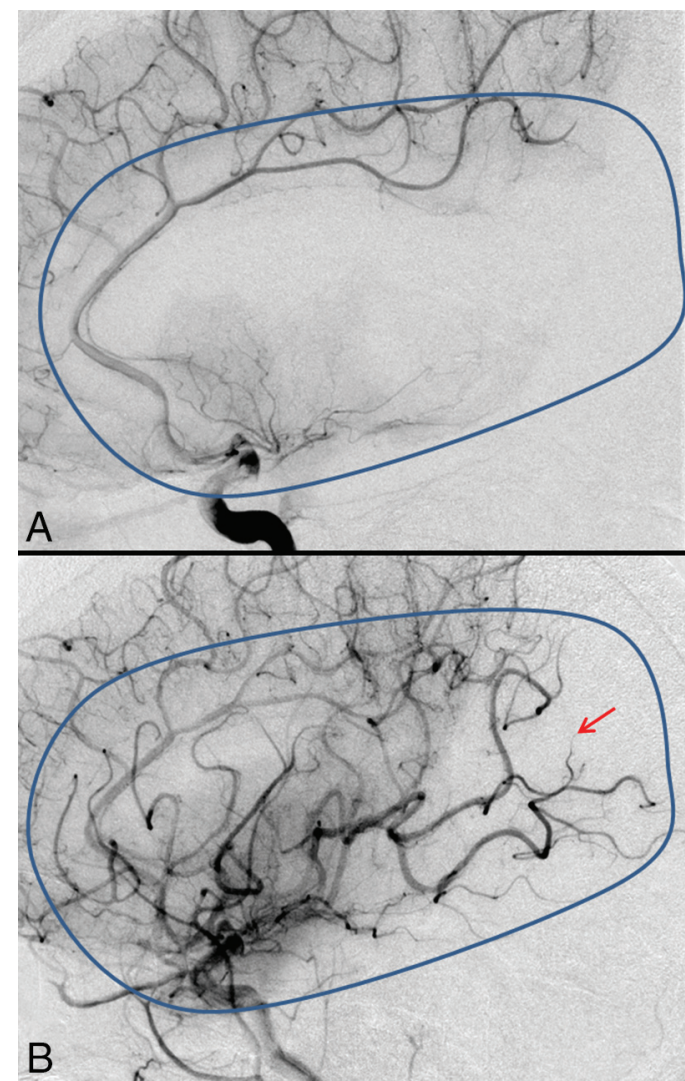

FIG 3. Measuring the extent of revascularization. Comparison between the $\mathrm{TICl}$ scoring before ( $\mathrm{TICI}$, $A$ ) and after ( $\mathrm{TICI} 2 \mathrm{~b}, B$ ) mechanical thrombectomy in the late arterial phase. The blue outline depicts the normal extent of the MCA territory. Note, the region shown by the red arrow suggesting non-/slow filling of a few distal cortical branches.

inner catheter for arch access, and a stent retriever $(4 \times 40 \mathrm{~mm}$ for M1 occlusions) with an appropriate microcatheter (this may change with advances in technology). The angiography staff (technicians and nurses) is cross-trained to be capable of managing work-flow seamlessly, single-handed if such a situation arises. It is not advisable to delay the procedure for shaving the groin region or insertion of a Foley catheter. ${ }^{21}$

18:14. Groin puncture was achieved, and an $8 \mathrm{~F}$ femoral sheath was inserted in the right common femoral artery. Meanwhile, the balloon-guided catheter was taken over a $5.5 \mathrm{~F}$ inner catheter in a coaxial fashion (the shape of the coaxial catheter is decided on the basis of the coronal formats of the CT angiogram of the arch and neck vessel; no exchange wire is used) and was parked in the internal carotid artery. We do not recommend time-consuming anatomic and collateral assessment during angiography because this information is already available from the CTA.

18:19. The first intracranial angiography run confirmed the left MCA segment 1 occlusion with an initial TICI flow of zero (no perfusion, no antegrade flow beyond the point of occlusion) (Fig $3 A)$.

\section{Neurointervention: Deciding on Devices and Techniques}

Many endovascular techniques are available for thrombus removal like intra-arterial thrombolysis, thrombus aspiration, stent retrievers, wire disruption, and so forth. However, all the recently published endovascular trials used newer retrievable stents in most of their patients $(81.5 \%, 86.1 \%, 89 \%, 100 \%$, and $95.1 \%$ in MR CLEAN, ESCAPE, SWIFT-PRIME, EXTEND-IA, and REVASCAT, respectively). Newer stent retrievers like the Solitaire FR (Covidien, Irvine, California) and Trevo (Stryker, Kalamazoo, Michigan) have been proved superior in efficacy and safety compared with the first-generation Merci retrievers (Concentric Medical, Mountain View, California). ${ }^{22}$ ESCAPE and SWIFT-PRIME trialists also advocated for the use of negative suction through a balloon-guide catheter with proximal balloon occlusion during thrombus retrieval to avoid distal emboli. ${ }^{2,4}$

18:23. A .021-inch microcatheter was taken over a .016-inch microguidewire (with the tip shaped to give a nontraumatic $J$ configuration) into the occluded MCA and was carefully navigated distal to the site of the thrombus (proximal M2 segment). A check microcatheter injection with minimal contrast was performed to confirm the correct positioning of the distal end of the microcatheter and to rule out any iatrogenic microguidewire perforations. An appropriately sized stent retriever $(4 \times 40 \mathrm{~mm}$ as default) was then carefully deployed across the M1 segment. After the deployment of the stent retriever, an angiographic run was performed primarily to assess the immediate bypass effect after stent deployment. In case there is absolutely no bypass effect, the possibilities are the following: 1) The stent retriever is not in the correct position and is covering only part of the clot. The exact location of the clot/thrombus is relatively easy to determine on the source images of the multiphase CTA: The proximal end of the clot can be determined on the first phase while the distal end of the clot can be seen on the second or third phase. In this case, without waiting further, the stent retriever is repositioned. 2) There is complete capture of the clot: The clot has come through the interstices of the stent and is ready to be pulled out. 3) The clot is firm and the stent retriever has no impact on the clot. The differentiation between 2 and 3 is difficult and often the only way to differentiate is to actually remove the stent and see if the clot is captured. In case there is some degree of bypass effect, one can wait 2-3 minutes and repeat a run; as the clot starts to get incorporated into the stent retriever, the repeat run shows irregularity and narrowing of the lumen of the vessel. This is usually a sign that the thrombus has been incorporated and can be removed.

In addition, if there is excellent flow due to a temporary bypass effect, it is important to remember that at that time the brain is getting excellent blood flow and can theoretically "reset the clock" in case the vessel reoccludes after removal of the stent retriever.

18:25. After retrieval of the stent, a control left ICA angiogram demonstrated restoration of flow in both the M1 and both M2 branches with residual occlusion of the distal M2 branch (Fig $3 B)$. The patient started improving clinically on the angiography table. The patient was assessed and the NIHSS was recorded. As soon as reperfusion was achieved, the stroke physician ensured that the blood pressure was lowered. The patient was transferred to the neurology critical care unit for further care. 


\section{Neurointervention: Assessing the Neuroangiographic Outcome}

Success of endovascular therapy can be judged by measuring reperfusion by using the TICI or the modified TICI scoring system. ${ }^{23}$ Generally speaking, a TICI score of $2 \mathrm{~b}$ or 3 is considered good reperfusion. Recently, a revised TICI scale that includes a $2 \mathrm{c}$ designation has been advocated for better end-point assessment. ${ }^{24}$ The reperfusion rates in the recent trials (TICI or modified TICI) were $72.4 \%, 58.7 \%, 86.2 \%, 88 \%$, and $65.7 \%$ in ESCAPE, MR CLEAN, SWIFT-PRIME, EXTEND-IA, and REVASCAT, respectively. ${ }^{1-5}$ The reasons for this variability are currently not clear and may be related to lack of standardized methodology for scoring and patient selection.

\section{Neurointervention: Deciding on Tandem Lesion Treatment}

The implications of emergency treatment of additional proximal extracerebral artery disease on the outcome have yet to be studied; $12.7 \%, 32.2 \%$, and $18.6 \%$ of patients in the intervention group in the ESCAPE, MR CLEAN, and REVASCAT trials, respectively, had documented ipsilateral cervical arterial occlusive disease on angiography; and $12.9 \%$ of patients in the MR CLEAN trial and $8.7 \%$ in the REVASCAT trial underwent cervical artery stent placement in the acute setting. ${ }^{1,2,5}$ In ESCAPE, the use of acute cervical carotid stent placement was discouraged. The best way of treating cervical carotid disease in acute stroke is still unclear, and further post hoc analysis of these major trials will hopefully give more answers.

In-Hospital Follow-Up. The next day following admission, the patient's NIHSS score was zero, and he was symptom-free. The follow-up DWI showed a small lesion in his caudate nucleus. His echocardiogram revealed severe dilative cardiomyopathy with presumed intracardiac thrombus as the source of his embolization. The patient was discharged home on warfarin (Coumadin).

Ninety-Day Clinic Follow-Up. After 3 months, the patient's NIHSS score was still zero; his Barthel-Index score of 100 and mRs score of zero indicated that he was fully functional and independent.

\section{CONCLUSIONS}

In patients with anterior circulation acute ischemic stroke due to large-vessel occlusion, endovascular treatment by using stent retrievers is now the standard of care. While the precise imaging paradigm was slightly variable across trials, there is clear evidence to support the use of CT/CTA as part of the work-up. We routinely use multiphase CTA to get additional information on collaterals and use it to further refine the ASPECTS reading. Additional imaging may be performed, such as CTP, as long as it does not introduce delays and does not falsely exclude patients from the benefit of endovascular therapy. Future focus should be on integrating such techniques into the stroke workflow. Several factors of endovascular workflow need to be addressed within the near future: The 3 most important, in our opinion, are geographic access to centers with angiography suites, prenotification, and parallel processing with a multispecialty team approach.

\section{ACKNOWLEDGMENTS}

We thank all the members of the Calgary Stroke Program who participated in the care of this patient.

Disclosures: Mayank Goyal—RELATED: Grant: Covidien, * Comments: partial funding of the ESCAPE trial; Consulting Fee or Honorarium: Covidien, Comments: for speaking engagements; for design and conduct of the SWIFT-PRIME trial; Other: GE Healthcare, Comments: licensing agreement for further development of systems of acute stroke diagnosis; Statement: M.G. was one of the Principal Investigators for the ESCAPE and SWIFT-PRIME trials; he was also the core lab lead for cross-sectional imaging within the REVASCAT study. He has a consulting agreement with Covidien for the conduct and design of SWIFT-PRIME and for teaching engagements. Covidien also provided part of the funding for the ESCAPE trial through a grant to the University of Calgary. M.G. has a licensing agreement with GE Healthcare for systems of stroke diagnosis. Bijoy K. Menon-Statement: B.K.M. was a member of the steering and executive committee of the ESCAPE trial and Principal Investigator for PRovelT (PeRfusion CT using the Aquilion One: Visual assessment of blood flow parameters in a clinical setting Enhances diagnostic and prognostic ability in patients with ischemic strokes when compared to NCCT and CTA based Imaging Techniques) study testing the utility of multiphase CTA and other imaging modalities in the triage of patients with stroke. B.K.M. holds a Heart and Stroke Foundation/University of Calgary Professorship in Stroke Imaging and a Canadian Institutes of Health Research New Investigator Award. *Money paid to the institution.

\section{REFERENCES}

1. Berkhemer OA, Fransen PSS, Beumer D, et al; for the MR CLEAN Investigators. A randomized trial of intraarterial treatment for acute ischemic stroke. $N$ Engl J Med 2015;372:11-20 CrossRef Medline

2. Goyal M, Demchuk AM, Menon BK, et al; for the ESCAPE Trial Investigators. Randomized assessment of rapid endovascular treatment of ischemic stroke. N Engl J Med 2015;372:1019-30 CrossRef Medline

3. Campbell BC, Mitchell PJ, Kleinig TJ, et al; for the EXTEND-IA Investigators. Endovascular therapy for ischemic stroke with perfusion-imaging selection. $N$ Engl J Med 2015;372:1009-18 CrossRef Medline

4. Saver JL, Goyal M, Bonafe A, et al; for the SWIFT-PRIME Investigators. Stent-retriever thrombectomy after intravenous t-PA vs. t-PA alone in stroke. N Engl J Med 2015;372:2285-95 CrossRef Medline

5. Jovin TG, Chamorro A, Cobo E, et al; for the REVASCAT Trial Investigators. Thrombectomy within $\mathbf{8}$ hours after symptom onset in ischemic stroke. N Engl J Med 2015;372:2296-306 CrossRef Medline

6. National Institute of Neurological Disorders and Stroke. Proceedings of a National Symposium on Rapid Identification and Treatment of Acute Stroke. http://www.ninds.nih.gov/news_and_events/proceedings/ strokeworkshop.htm. Accessed March 20, 2015

7. Kamal N, Benavente $\mathrm{O}$, Boyle $\mathrm{K}$, et al. Good is not good enough: the benchmark stroke door-to-needle time should be $\mathbf{3 0}$ minutes. Can J Neurol Sci 2014;41:694-96 CrossRef Medline

8. Prabhakaran S, Ward E, John S, et al. Transfer delay is a major factor limiting the use of intra-arterial treatment in acute ischemic stroke. Stroke 2011;42:1626-30 CrossRef Medline

9. El Khoury R, Jung R, Nanda A, et al. Overview of key factors in improving access to acute stroke care. Neurology 2012;79:S26-34 CrossRef Medline

10. Menon BK, Saver JL, Goyal M, et al. Trends in endovascular therapy and clinical outcomes within the nationwide Get With The Guidelines-Stroke Registry. Stroke 2015;46:989-95 CrossRef Medline

11. Goyal M, Menon BK, Hill MD, et al. Consistently achieving computed tomography to endovascular recanalization $<90$ minutes: solutions and innovations. Stroke 2014;45:e252-56 CrossRef Medline

12. Menon BK, d'Esterre CD, Qazi EM, et al. Multiphase CT angiography: a new tool for the imaging triage of patients with acute ischemic stroke. Radiology 2015;275:510-20 CrossRef Medline

13. Menon BK, Campbell BC, Levi C, et al. Role of imaging in current acute ischemic stroke workflow for endovascular therapy. Stroke 2015;46:1453-61 CrossRef Medline 
14. Zussman B, Flanders A, Rosenwasswer MD, et al. Behind the technology: CT perfusion in the setting of acute stroke management. JHN Journal 2010;5. http://jdc.jefferson.edu/jhnj/vol5/iss2/1. Accessed September 20, 2015

15. Campbell BC, Yassi N, Ma H, et al. Imaging selection in ischemic stroke: feasibility of automated CT-perfusion analysis. Int J Stroke 2015;10:51-54 CrossRef Medline

16. Goyal M, Fargen KM, Menon BK. Acute stroke: Bayes' theorem and the art and science of emergency decision-making. J Neurointerv Surg 2014;6:256-59 CrossRef Medline

17. Almekhlafi MA, Eesa M, Menon BK, et al. Ultrashort imaging to reperfusion time interval arrests core expansion in endovascular therapy for acute ischemic stroke. J Neurointerv Surg 2013;5(suppl 1):i58-61 CrossRef Medline

18. Fonarow GC, Smith EE, Saver JL, et al. Improving door-to-needle times in acute ischemic stroke: the design and rationale for the American Heart Association/American Stroke Association's target: stroke initiative. Stroke 2011;42:2983-99 CrossRef Medline

19. Abou-Chebl A, Lin R, Shazam Hussain M, et al. Conscious sedation versus general anesthesia during endovascular therapy for acute anterior circulation stroke: preliminary results from a retrospective, multicenter study. Stroke 2010;41:1175-79 CrossRef Medline

20. Almekhlafi MA, Hockley A, Desai JA, et al. Overcoming the evening/ weekend effects on time delays and outcomes of endovascular stroke therapy: the Calgary Stroke Program experience. J Neurointerv Surg 2014;6:729-32 CrossRef Medline

21. Nedeltchev K, Arnold M, Brekenfeld C, et al. Pre- and in-hospital delays from stroke onset to intra-arterial thrombolysis. Stroke 2003; 34:1230-34 CrossRef Medline

22. Nogueira RG, Lutsep HM, Gupta R, et al; for the TREVO 2 Trialists. Trevo versus Merci retrievers for thrombectomy revascularisation of large vessel occlusions in acute ischemic stroke (TREVO 2): a randomised trial. Lancet 2012;380:1231-40 CrossRef Medline

23. Fugate JE, Klunder AM, Kallmes DF. What is meant by "TICI"? AJNR Am J Neuroradiol 2013;34:1792-97 CrossRef Medline

24. Goyal M, Fargen KM, Turk AS, et al. 2C or not 2C: defining an improved revascularization grading scale and the need for standardization of angiographic outcomes in stroke trials. J Neurointerv Surg 2014;6:83-86 CrossRef Medline 